acetone $(12,10,8,6$, and $5 \%)$ to pure water, a destruction

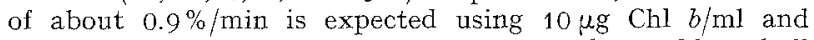
$30 \mathrm{ppm} \mathrm{O}_{3}$, indicating that the ozone attack on chlorophyll could occur in an aqueous environment. Since the only difference between the two forms of chlorophyll used here is the replacement of a methyl group on pyrrole ring II of the porphyrin in $\mathrm{Chl} a$ by a formyl group in $\mathrm{Chl} b$, the site of ozone attack may be in ring II, at least for $\mathrm{Chl} b$. The preferential oxidation of $\mathrm{Chl} b$ by ozone might help prevent destruction of Chl a in vivo.

Supported by U.S. Public Health Service Research Grant GM 15183 from the National Institute of General Medical Sciences.

Received December 10, 1973

1. De Koning, H. W., Jegier, Z.: Atmos. Environ. 2, 615 (1968)

2. Nobel, P. S.: Plant Physiol. 42, 1389 (1967)

3. Nobel, P. S., Wang, C.-T.: Arch. Biochem. Biophys. 157, $388(1973)$

4. MacKinney, G.: J. Biol. Chem. 140, 315 (1941)

5. Vernon, L. P., Seely, G. R.: The Chlorophylls. New York: Academic Press 1966

\section{Eliminierung von Pentachlorphenol durch Pflanzen aus Gewässern}

Käthe Seidel

Max-Planck-Institut für Züchtungsforschung

Limnologische Arbeitsgruppe, Ktefeld-Hülserberg

Pentachlorphenol (PCP) ist ein starkes Gift für Bakterien, Schwämme, Algen, Pilze und Insekten. Nach Liebmann [1] liegt in Gewässern die Letalitätsgrenze für Karpfen bei $5 \mathrm{mg} / 1$ (3-4h Einwirkungszeit). Als Schädlichkeitsgrenze für Fische im allgemeinen gibt der Autor $0,2-0,45 \mathrm{mg} / \mathrm{l}$ an.

Uns interessierte, wie höhere Pflanzen auf dieses halogenierte Phenol reagieren. Es wurden zunächst Pflanzen verwendet, die "Binsen-Charakter" haben, d.h. mit großem Aerenchym ausgerüstet sind und basales Halmwachstum zeigen: Juncus effusus L., Juncus maritimus Lamk., Bolboschoenus maritimus (L.) Palla, Schoenoplectus lacustris (L.) Palla und Schoenoplectus tabernaemontani (C. C. Gmel.) Palla. Juncus effusus (Flatterbinse) war besser geeignet als Juncus maritimus (Meerbinse). Ungeeignet waren Schoenoplectus lacustris (Flechtbinse) und Schoenoplectus tabernaemontani (Salzbinse) sowie Bolboschoenus maritimus (Meerstrandbinse).

Die Versuchsbecken wurden gegen die Umgebung abgedichtet und mit inertem, teilchenförmigem Material (vorzugsweise Kies mit bestimmter Korngröße) gefüllt, und zwar so hoch, daß sich die Wurzeln der Versuchspflanzen auch vertikal gut ausbreiten konnten. Das durchfließende, mit PCP belastete Wasser überspülte nicht die Oberfläche des Kieses, d.h. die Halme kamen nicht oder kaum mit der Pentachlorphenoliösung in Berührung. Die Versuche dauerten 2 Jahre. Die Pflanzen blieben ungeschädigt. Die Belastung betrug $3 \mathrm{mg} / \mathrm{l}$, die Fließgeschwindigkeit war so eingestellt, daß bei $1 \mathrm{~m}^{2}$ bepflanzter Fläche $960 \mathrm{mg}$ PCP pro Tag entfernt werden konnten.

Häufig hat mit PCP belastetes Wasser einen hohen Kochsalzgehalt. Ein Industrieabwasser mit $4 \mathrm{mg} \mathrm{C}_{6} \mathrm{Cl}_{5} \mathrm{OH}$ und $20 \mathrm{~g}$ $\mathrm{NaCl} / \mathrm{l}$ wurde durch mit Juncus effusus bepflanzte Kiesbecken geleitet, nachdem sich die Pflanzen an PCP adaptiert hatten. Nach $3 \mathrm{~h}$ wurden $2 \mathrm{~h}$ lang mit unbelastetem Wasser von ungefähr $+15^{\circ} \mathrm{C}$ die gebildeten Salzkristalle aufgelöst und abgespült. Es folgte wieder ein dreistündiger Durchlauf von belastetem Wasser, dem sich eine 16stündige Spülung anschloß. Abwasser und Spülwasser wurden in einem Sammelbecken aufgefangen und nach einem vollständigen Tageszyklus abgelassen. Dieses Mischwasser war fast immer frei von Pentachlorphenol und enthielt 3,5-5,0 g NaCl/l. Die pro $\mathrm{m}^{2}$ bepflanzter Fläche und Tag entfernte Menge an PCP betrug $640 \mathrm{mg}$. Der Versuch dauerte ein Jahr. Auch hier blieben die Pflanzen gesund und wüchsig. Um den Mechanismus aufzuklären, der die Pflanzen zu dieser Leistung befähigt, bedarf es noch weiterer grundlegender Untersuchungen.

Eingegangen am 27. November 1973

1. Liebmann, H.: Handbuch der Frisch- und Abwasserbiologie II. München: R. Oldenbourg 1958

\section{Schoenoplectus lacustris (L.) Palla zur Reinigung von Gichtwässern}

Käthe Seidel

Limnologische Arbeitsgruppe Dr. Seidel

am Max-Planck-Institut für Züchtungsforschung,

Krefeld-Hülserberg

Herta Boden

Stahlkombinat Resita, Rumänien

\section{E. Lörinczi}

Timisoara, Rumänien

Die Frage lautete, ob Cyan-Verbindungen, für Menschen und Tiere als giftig bekannt, auch für höhere Pflanzen schädlich sind und ob evtl. bestimmte Pflanzenarten solche Verbindungen eliminieren können. Bei der Auswahl der Versuchspflanzen gingen wir weiter von der Frage aus, ob Pflanzen, die nach unseren Erfahrungen $\mathrm{CH}-$ Verbindungen aufnehmen, aufbrechen und in ihren Stoffwechsel einbauen, auch CN-Verbindungen metabolisieren können. Aus diesem Pflanzensortiment wählten wir Schoenoplectus lacustris (L.) Palla und führten 1966 erste Messungen mit Gichtwässern eines niederrheinischen Stahlwerkes durch, das uns auch Analysenhilfe gab. Cyanid-haltige Abwässer entstehen in der Stahlindustrie und werden besonders dort gefährlich, wo sie anschließend Wässer belasten, die zur Trinkwasserversorgung verwendet werden müssen. Dies war der AnlaB, im Stahlkombinat Resita. in Rumänien eine Versuchsanlage aufzubauen. Während in den vorhandenen Absatzteichen ein Reinigungsgrad bei Cyaniden von $32,0 \%$, bei Rhodaniden von $0,0 \%$ erzielt wird, betrug 1968 in der Versuchsanlage der Reinigungsgrad bei Cyaniden + Rhodaniden:

\begin{tabular}{lllll}
\hline $\begin{array}{l}\text { Aufenthaltszeit } \\
2-4 \mathrm{~h}\end{array}$ & & & & \\
$\begin{array}{l}\text { Sommer } \\
\end{array}$ & Winter & & Sommer & Winter \\
\hline $66,6 \%$ & $27,7 \%$ & & $100 \%$ & $62,6 \%$ \\
\hline
\end{tabular}

Die bepflanzte Anlage wurde nach einem bestimmten, 1973 patentierten System aufgebaut.

Zur Zeit laufen Untersuchungen über den Verbleib der CyanVerbindungen, z. B. Abgabe an die Atmosphäre, Akkumulation und Metabolisation bei Mikro- und Makrophyten.

Eingegangen am 27. November 1973

\section{Lauterzeugungsmechanismus und Lautrezeption von Kleinzikaden}

A. Haße

Freie Universität Berlin,

Institut für Allgemeine Zoologie und experimentelle Morphologie

Das Lautverhalten der Gattung Euscelis (Homoptera: Cicadina) ist für verschiedene Arten beschrieben worden [1]. Die Lautäußerungen beider Geschlechter dienen dem Zusammenfinden der Partner.

Die Lautabgabe erfolgt bei Euscelis nicht über , Schallplatten" durch Abstrahlung von Luftschall wie bei Singzikaden. Die durch die paarigen Schallmuskeln erzeugte Energie wird vom Erregersystem (1. und 2. abdominales Tergum) auf den Vibrationserzeuger, dargestellt durch weitere abdominale Terga, in kurzen Stößen übertragen. Sie bauen eine Nahfeldströmung auf [2]. Die Konstituenten-Summation der relativen spektralen Frequenzanteile zeigt, daß die kurze, periodische Stoßerregung die schwingungsfähigen Teile $z u$ einheitlichen Schwingungen veranlaßt, wobei systembestimmte Frequenzanteile in stärkerem Maße an das Medium abgegeben werden.

Aus physikalischen Grüaden sind die Tiere als kleine Punktstrahler bei den verwendeten Frequenzen (wesentliche Maxima zwischen 150 und $1500 \mathrm{~Hz}$ ) zur Abstrahlung von sich periodisch fortpflanzendem Luftschall nicht geeignet; es kann kein zur Luftschallverständigung geeignetes Fernfeld aufgebaut wer- 